\title{
Selenoproteins - biochemistry and clinical relevance
}

Five decades ago, selenium had been recognized as an essential trace element for bacteria by Jane Pinsent and for higher vertebrates by Klaus Schwarz (see photo) and Calvin M. Foltz, who isolated a selenium-containing compound from hog kidney, the 'factor 3' that protected rats against dietary liver necrosis. Fifteen years later, the first selenoproteins were detected in mammals as well as in bacteria indicating that the trace element might fulfil its biological function as an integral constituent of enzymes. In 2000, the German Research Council (Deutsche Forschungsgemeinschaft, DFG) positively evaluated and subsequently funded the Priority Program 1087 (Schwerpunktprogramm) focusing on basic, applied and clinically oriented research on selenoproteins, their biochemistry and function. The current issue of Biological Chemistry presents state-of-the-art reviews and original research communications written by scientists who were directly or indirectly supported by the Priority Program. This special issue is expected to provide a topical view of the frontiers of selenoprotein biochemistry and its clinical relevance.

Selenoproteins represent a new class of peculiar redox-active proteins containing the 21 st proteinogenic amino acid, selenocysteine (Sec), in their active center. Selenium research has been significantly boosted by the

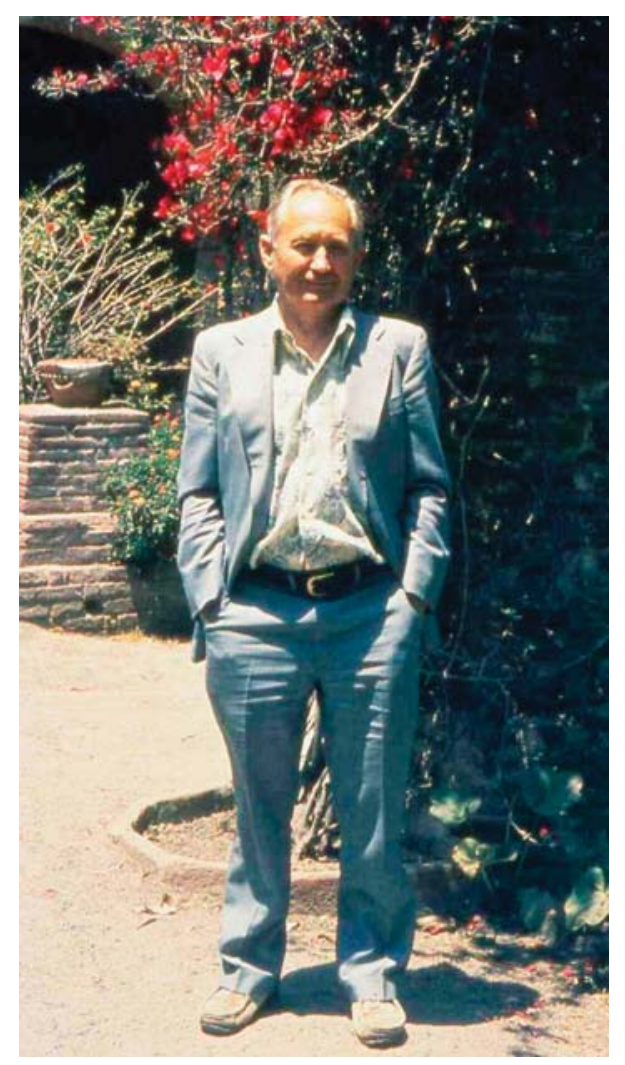

Klaus Schwarz (1914-1978), a pioneer of biomedical selenium research. expansion of the genetic code by two more codons: UAG for pyrrolysine and UGA encoding selenocysteine. Briefly, UGA, the opal stop codon, can be cotranslationally reinterpreted as incorporation signal for selenocysteine, which is the selenium homolog of cysteine, if further requirements are met: (i) a context structural feature of the selenoprotein mRNA, the selenocysteine insertion sequence (SECIS) which is located in the 3'UTR in eukaryotes and not immediately downstream of the UGA codon, as initially detected in prokaryotes; (ii) specific translation factors that recognize the latter in a seleniumdependent manner; (iii) a Sec-loaded specific tRNA ${ }^{\mathrm{Sec}}$ to incorporate selenocysteine into the nascent protein chain; and (iv) the machinery to synthesize and charge this tRNA. Altogether, a whole set of proteins is required to specifically insert selenocysteine into the elongating peptide chain and to suppress termination. Several of the key factors required have only recently been identified and now help to advance this flourishing research field.

A further major boost came to the selenium field in 2003, when the human selenogenome was identified by novel algorithms that safely identified combinations of UGA codons and SECIS hairpin loops that follow specific folding patterns and expose key bases for recognition by SECIS binding proteins. The human selenogenome comprises 25 genes which at least in part are translated into different proteins with distinct functions. With this knowledge, the biomedical relevance of selenium can no longer be simply addressed by popular terms, such as 'antioxidative defence' or 'protection from oxidative stress'. Not even the fact that 5 out of the 25 genes encode peroxidases justifies this simplification, because some of the glutathione peroxidases have evidently adopted surprising roles, such as dampening the generation of inflammatory mediators, regulating the insulin response or assuring spermiogenesis. All three known thyroid hormone deiodinases, more recently identified to be selenoproteins, are involved in thyroid hormone biosynthesis and metabolism. The three thioredoxin reductases are linked to hydroperoxide metabolism via the peroxiredoxins, and thus involved in both intracellular and extracellular redox control, but they are also pivotal to the biosynthesis of deoxynucleotides. New horizons are opened up by deletions or mutational alterations of selenoproteins or proteins involved in selenoprotein biosynthesis. For example, the rigid spine syndrome proved to be associated with mutations of selenoprotein $\mathrm{N}$, and impairment of growth, development and thyroid hormone feedback regulation are linked to an impaired function of SECIS binding protein 2. Fortunately, the number of selenoprotein-encoding genes is so low that the alphabet was just sufficient to provide letters for the selenoproteins with unknown functions such as Sel-H, I, K, M, N, $\mathrm{O}, \mathrm{R}, \mathrm{S}, \mathrm{T}, \mathrm{V}, \mathrm{W}$, but it is large enough to forecast future surprises. 
The elucidation of the biology of selenium gained further momentum with the successful application of transgenic technology. Knockout and conditional knockout models and transgenic expression of selenoproteins helped to advance the field and to illustrate that several of the selenoproteins already play a critical role during mammalian development, e.g., thioredoxin reductases and one of the glutathione peroxidases, thus explaining why a complete knockout of the mouse trsp (tRNA ${ }^{\mathrm{Sec}}$ ) is embryonically lethal. Inverse genetics also revealed that selenium biochemistry is indispensible for appropriate brain function, an aspect that had not become obvious from nutritional selenium deprivation.

In the meantime, selenogenomes of various species have been characterized, i.e., those of important human pathogens. Their functional analysis is still rudimentary. However, they reveal that the use of the trace element changed dramatically during evolution. The machinery for selenoprotein biosynthesis being lost in yeast and terrestric plants, it is otherwise conserved from archaea and bacteria up to protozoa and higher animals, although with marked differences. Furthermore, selenoprotein families are not regularly conserved between related species and rarely between domains, kingdoms and phyla. Certainly, this diversification must be seen as a major obstacle in any attempt to deduce the function, e.g., of a human protein by studying well-investigated but genetically remote model organisms. On the other hand, the chance to selectively target the unrelated selenoproteins of pathogenic microorganisms is being discussed as a challenging therapeutic option.

The Priority Program markedly supported selenoprotein research in Germany based on long-standing tradition and work of pioneers in the field, who initially were instrumental and successful in convincing the national and later on the international review board of this program - first, to install it and then to grant two prolongations. Novel scientific and clinical relevant information has been obtained, e.g., illustrating the role of selenium in development, male fertility, carcinogenesis, endocrinology and stem cell research. In addition, the program advanced research to target drugs to selenocysteineactive sites of proteins, assisted by modeling and structure analyses of some selenoproteins. Novel bioinformatic tools and combinatorial chemistry programs were successfully implemented, which helped to create a high interdisciplinary technology-advancing and communication-fostering program. At the end of the funding period, intense collaborations between the funded teams, strong international networks, and even collaborations with joint publications between former or current reviewers or scientists invited to the program workshops have been established and are partially documented here.

The DFG, its advisory bodies and review boards are thanked for taking the initiative to foster a rapidly emerging field just in time. This facilitated the recruitment and education of many young scientists and their introduction into the field, and thereby effected a smooth change of researchers in our community. This program also markedly helped to blow away some 'odd flavor' from the field, which definitely is not a paramedic one but hardcore science and biochemistry with all its methodological and conceptual facets. Some selenium compounds, such as $\mathrm{H}_{2} \mathrm{Se}$ and its methylated derivatives still smell, but others are essential for human and animal health, disease prevention and even therapy.

The editors of this highlight issue hope that you enjoy the compiled scientific selenium-related papers, some of them being mini-reviews, some being original work or a combination of both - all critically peer-reviewed according to the procedures of Biological Chemistry. We would like to thank all authors, their teams, and the Editorial Board as well as the DFG for this opportunity to present an update on the state-of-the-art in the selenoprotein field.

\section{August Böck}

Department Biologie 1 - Mikrobiologie

Universität München

Maria-Ward-Straße 1a

D-80638 München

Germany

e-mail: august.boeck@|rz.uni-muenchen.de

Leopold Flohé

MOLISA GmbH

Universitätsplatz 2

D-39106 Magdeburg

Germany

e-mail: L.Flohe@t-online.de

\section{Josef Köhrle}

Institut für Experimentelle Endokrinologie

Campus Charité Mitte

Charité Universitätsmedizin Berlin

Humboldt-Universität zu Berlin

Charitéplatz 1

D-10117 Berlin

Germany

e-mail: Josef.Koehrle@charite.de 